\title{
CAN SMALL RARE PREY BE CHEMICALLY DEFENDED? THE CASE FOR MARINE LARVAE'
}

\author{
NiEls Lindquist and Mark E. Hay \\ University of North Carolina at Chapel Hill, Institute of Marine Sciences, \\ Morehead Citv, North Carolina 28557 USA
}

\begin{abstract}
Marine larvae are consumed by a wide variety of generalist fishes and particle-feeding invertebrates, but larvae of any particular species probably constitute a small and variable portion of the diet for these consumers. Because virtually all consumers can ingest small quantities of noxious compounds with minimal detrimental effects, it is uncertain that toxic chemicals in larvae could be consumed in quantities sufficient to select for predator recognition and avoidance. Despite this, chemically defended larvae do occur. We show that, at low doses, secondary metabolites (the didemnins) from adults and larvae of the Caribbean tunicate Trididemnum solidum induced vomiting in fish, resulting in rapid learned aversion to the didemnin-defended food. The particle-feeding anemone Aiptasia pallida did not learn to avoid the chemically defended food. When anemones ingested the chemical equivalent of 15 larvae/d, representing $<2 \%$ of the mass of their total daily diet, the didemnins in the "larvae" significantly reduced: (1) growth of adults by $82 \%$, (2) combined growth of adults and daughter clones by $76 \%$, (3) production of daughter clones by $44 \%$, and (4) average mass of individual daughter clones by $41 \%$. At higher water temperatures, anemones cloned more rapidly, but the negative effects of consuming didemnins also occurred more rapidly. Significant differences in the number of daughter clones produced between treatment and control anemones occurred after only $4 \mathrm{~d}$ at seawater temperatures of $27^{\circ}-29^{\circ} \mathrm{C}$ vs. $32 \mathrm{~d}$ at seawater temperatures of $18^{\circ}-21^{\circ} \mathrm{C}$. Thus consumption of even very small quantities of secondary metabolites can decrease consumer fitness substantially and select for predators that recognize and avoid chemically defended larvae, as do many consumers that co-occur with Trididemnum solidum larvae. This is the first rigorous demonstration that consumption of marine secondary metabolites can decrease consumer fitness when ingested at ecologically realistic doses.
\end{abstract}

Key words: chemical defenses; fitness; larvae; learning; physiological effects; predator-prey interactions; reproduction; secondary metabolites.

\section{INTRODUCTION}

The advantages of chemically repelling consumers are obvious and have been studied extensively in both marine and terrestrial communities (see reviews in Paul 1992 and Rosenthal and Berenbaum 1992, respectively). In contrast, relatively few studies assess how consuming ecologically realistic amounts of chemically defended prey will impact the fitness of marine consumers and thus the evolution of effective chemical defenses in marine prey. Although it seems reasonable to assume that consumers avoid prey containing certain secondary metabolites because the metabolites negatively affect consumer fitness, available data suggest that numerous deterrent plant metabolites have no negative biological effects when consumed by herbivorous insects (Bernays and Chapman 1987, Bernays and Cornelius 1992). Additionally, there often is a poor correlation between the toxicity of secondary metabolites (when injected into or rubbed onto insects, or dispersed in seawater containing marine consumers) and how they affect feeding by ecologically relevant consumers

\footnotetext{
' Manuscript received 3 January 1994; revised 7 June 1994; accepted 8 September 1994; final version received 17 October 1994.
}

(Bernays and Chapman 1987, Hay et al. 1987, Hay and Fenical 1988, Bernays 1991, Pawlik 1993).

Numerous studies assessing the performance of insects feeding on plants containing deterrent compounds or artificial foods into which these compounds have been incorporated have demonstrated decreased growth or survivorship on these diets. Few, however, have demonstrated that the changes in growth or survivorship result from physiological effects of the compounds rather than from reduced feeding when consumers are restricted to chemically repugnant foods (see reviews by Feeny 1992 and Slansky 1992). Thus, even for relatively well-studied cases of chemically mediated plant-insect interactions, it is often unclear whether consumers limit ingestion of chemically rich prey due to their negative postingestive effects or whether prey are capitalizing on idiosyncrasies of consumer taste and producing compounds consumers find distasteful but that have little effect on their fitness.

Additionally, there is a problem with using most insect species as models of chemically deterred consumers because the majority of herbivorous insects are relatively specialized feeders, consuming only a few types of chemically or taxonomically related prey (Bernays 
1989). This degree of feeding specialization is rare among most other groups of consumers, especially marine consumers, almost all of which are broad generalists rarely feeding on one species over an extended period of time (Hay 1992, Hay and Steinberg 1992). Thus, relatively specialized insect herbivores might normally consume significant quantities of their host's secondary metabolites for much of their lifetime, while generalist consumers would rarely experience a high concentration, or sustained intake, of any specific compound. In fact, it has been suggested that generalized feeding strategies are advantageous in that they provide a way for consumers to avoid ingesting toxic doses of any particular secondary metabolite (Freeland and Janzen 1974).

Given that virtually all consumers produce enzymes that allow them to consume small quantities of noxious compounds with minimal detrimental effects (Brattsten 1992), it is not obvious that generalist marine consumers will commonly ingest enough of a particular defensive metabolite to significantly reduce their fitness and thus select for individuals that recognize and avoid prey producing these compounds. This could, in theory, make it difficult for effective chemical defenses to evolve in marine prey. An extreme example of this might be exemplified by larvae of marine invertebrates because they (1) are consumed by a wide variety of generalist fishes and invertebrates (reviewed by Young and Chia 1987), (2) are small relative to the size of planktivorous fishes and benthic particle feeders that consume them, and (3) are often dispersed in the water column so that larvae of any particular species would not commonly be an appreciable portion of a consumer's diet [because holoplanktonic and meroplanktonic crustaceans are often major prey items of generalized planktivores, larvae commonly represent only a small fraction of total prey mass in a consumer's gut (Zelickman et al. 1969, Emery 1973, Hobson and Chess 1976, Purcell 1981, Livingston 1984)]. However, chemically mediated rejection of larvae, particularly brooded larvae of sessile invertebrates, appears common (Lucas et al. 1979, Young and Bingham 1987, Lindquist et al. 1992; N. Lindquist, personal observation), suggesting that even small doses of larval chemical defenses might harm consumers enough to select for recognition and avoidance of these larvae.

Brooded larvae may be especially good candidates for chemical defenses because adults produce relatively small numbers of relatively large larvae, each of which may represent a substantial investment by the adult, and thus may be worth defending. This pattern for brooders contrasts with external fertilizers, which produce hundreds of thousands to millions of small larvae (Thorson 1950), each of which represents a minimal investment to the adult. Additionally, because brooders commonly release relatively few larvae daily over a reproductive period of months, to year-round (Thorson 1950, Reiswig 1973, Ayling 1980, van Duyl et al. 1981,
Olson 1983, Davis 1988, Ilan and Loya 1990, Stoner 1990), these larvae will rarely be abundant enough to make up an appreciable part of any consumer's diet. In order for these larvae to be chemically defended they would thus have to make compounds that significantly decrease consumer fitness at very low doses, or make compounds with a taste that envokes behavioral rejection of the larvae as food even though the compounds might have no effect on consumer fitness.

We tested these alternative possibilities by periodically feeding two generalist consumers (the pinfish Lagodon rhomboides and sea anemone Aiptasia pallida) a "larval mimic" that contained natural concentrations of secondary metabolites (the didemnins) found in larvae and adults of the colonial Caribbean ascidian (sea squirt) Trididemnum solidum. Larvae of Trididemnum are (1) spawned during daylight hours (van Duyl et al. 1981), (2) large enough to be easily detected by fishes, and (3) swim too slowly to avoid capture; despite their vulnerability to predators, Trididemnum larvae are rejected as food by small reef fishes (Lindquist et al. 1992). Lindquist and co-workers (1992) demonstrated that at naturally occurring concentrations in larvae, as well as adults, the didemnins significantly deter fish feeding. Additionally, these metabolites may also explain the rejection of Trididemnum larvae by corals (van Duyl et al. 1981). Our "larval mimic" was not an exact chemical substitute for Trididemnum larvae (see Methods), but was intended to approximate the dose of secondary metabolites that a generalized consumer might ingest naturally when feeding on small rare prey, such as larvae.

To determine if periodic ingestion of small amounts of chemically defended prey could harm consumers, we conducted three feeding assays in which consumption of larval mimics was chemically similar to ingesting 15 Trididemnum larvae per day. This number of larvae was well below the number of ascidian larvae that may be ingested daily by benthic cnidarians in the field (Sebens and Koehl 1984). For the long-term assays with the sea anemone Aiptasia pallida our larval mimics accounted for $<2 \%$ of their daily food intake.

\section{Methods}

Determining the long-term effects for marine consumers of ingesting deterrent secondary metabolites has been difficult because generalist consumers that consistently co-occur with prey containing secondary metabolites commonly refuse to consume the compounds because of an innate recognition of (reviewed by Paul 1992, Pawlik 1993), or rapidly learned aversion to (Gerhart 1984, Young and Bingham 1987, Hay et al. 1987) the secondary metabolites even when they are offered in novel foods. Previous studies that document compound effects on consumer feeding, but rarely on their physiology, form the foundation for much of our current understanding of chemical defenses in marine plants and animals. These studies do 
not indicate why particular compounds are avoided by consumers. In contrast to the difficulties of getting generalists to eat treatment foods, consumers that selectively eat chemically rich prey may readily eat treatment foods; however, results from assays using these specialists will not apply to the vast majority of generalist marine consumers, and it is the generalists that appear most important in selecting for prey chemical defenses (Hay 1991, 1992, Hay and Steinberg 1992). Thus, a major problem associated with assessing longterm physiological effects of marine secondary metabolites on consumers has been finding an ecologically relevant consumer that will consistently eat treatment food and not learn to reject it during the course of an experiment.

\section{Feeding studies with fish}

Despite the learning ability of fishes (Kerfoot 1982, Gerhart 1984, Hay et al. 1987, Young and Bingham 1987), we attempted to identify the long-term physiological costs of consuming small quantities of the didemnins using the pinfish Lagodon rhomboides. Lagodon is an omnivorous fish ranging in the western Atlantic and the Caribbean Sea from New England to the Yucatan peninsula in Mexico (Darcy 1985). Although Lagodon and the ascidian producing the secondary metabolites we tested may overlap geographically in the northwest Caribbean, they rarely, if ever, occur in the same habitat; Lagodon is commonly found in protected inshore habitats (Darcy 1985) and Trididemnum solidum occurs on offshore coral reefs (Bak et al. 1981; N. Lindquist, personal observation). Additionally, because larvae of tropical colonial ascidians, including those of Trididemnum, usually settle within 15 min of spawning (reviewed by Svane and Young 1989, Lindquist et al. 1992), Trididemnum larvae are unlikely to be carried by currents to habitats where Lagodon is common. For this assay, we minimized the chance of prior consumer experience with didemnins by using Lagodon collected from temperate waters of North Carolina where the Caribbean ascidian is not known to occur.

Lagodon rhomboides (7-10 cm total length) were captured by seining through shallow seagrass beds near Harker's Island, North Carolina. Thirty fish were placed individually in perforated 1-L plastic bowls (hole diameter $=1 \mathrm{~cm}$, four holes per bowl) in a flow-through water table. Each of these bowls had a mesh bottom and sat on top of a smaller $(0.75 \mathrm{~L})$ plastic bowl. This set-up allowed us to recover, from the bottom container, any food items rejected by each fish. Fifteen fish that were fed didemnin-containing food were interspersed among 15 control fish that were fed the same food but without didemnins.

Each morning (0800-0900) for $10 \mathrm{~d}$ all fish were offered 10 pellets of an untreated commercial fish food (Hikari Cichlid Bio-Gold -"mini",-sized pellets) having a total dry mass of $168 \mathrm{mg}$. On days 6-10 at 1200-
1300 , all fish were also offered nine small food pellets (each $0.65 \mu \mathrm{L}=0.056 \mathrm{mg}$ dry mass) that fish could swallow whole; these pellets were made by pureeing together squid mantle flesh, an equal volume of distilled water, and sodium alginate at $2 \%$ of total wet mass. This liquid mixture was injected with a syringe into a $0.25 \mathrm{~mol} / \mathrm{L}$ solution of calcium chloride $\left(\mathrm{CaCl}_{2}\right)$ that caused the squid puree to form a gelatinous strand with a consistency much like that of cooked spaghetti. This strand could then be cut into standard-sized pellets (hereafter called "squid pellets"). Both the commercial fish food and the squid pellets were readily consumed by all fish. Each afternoon (1400-1500) for three consecutive days beginning on day 8 , control fish received two control squid pellets $(3.5 \mu \mathrm{L}=0.3 \mathrm{mg}$ dry mass each) without didemnins and treatment fish were offered a control squid pellet without didemnins followed several minutes later by a squid pellet that was identical to controls but also contained didemnins (i.e., a "larval mimic"). Because by day 10 a significant number of treatment fish would not ingest the "larval mimic," the experiment was terminated.

To make treatment squid pellets, a semipurified extract of Trididemnum solidum composed of $>95 \%$ didemnin cyclic peptides was mixed into the squid puree so that the combined volumetric concentration of didemnins in this food approximated that of Trididemnum larvae. Our "larval mimics" contained $7 \mu \mathrm{g}$ of didemnins $\mathrm{B}$, nor-B, D, E, M, and $\mathrm{X}$ at ratios of $\approx 10: 1: 4: 7$ : $1: 2$, respectively. Larvae of Trididemnum have a slightly lower total mass of didemnins $(\approx 12 \%$ less by volume) than do adults because larvae lack didemnins $M$ and $X$, however, the didemnins that are in larvae (didemnins B, nor-B, D, and E) occur at approximately those levels found in adults (Lindquist et al. 1992). Although the didemnin composition of our "larval mimic" did not exactly match that of Trididemnum larvae, total didemnin concentration was similar to natural levels and the methods achieved our goal of producing a food that mimicked the mass of secondary metabolites a generalized consumer might encounter by consuming a realistic number of larvae per day from a single prey species.

We recorded for each fish whether or not the two squid pellets offered each afternoon (1400-1500) were ingested or rejected. The undefended squid pellets offered to both treatment and control fish were always eaten except by one treatment fish on day 10. Significant differences in the consumption frequency of the second pellet offered to treatment and control fish were determined using the Fisher exact test. Preliminary feeding experiments showed that ingestion of didemnins causes Lagodon to regurgitate within 1-2 h; thus, $2 \mathrm{~h}$ after feeding control and treatment pellets to the fish, we checked the containers beneath each fish for regurgitated food. We roughly quantified the amount of food regurgitated at 1700 on days $8-10$ by counting the number of small pellets (from the 1200-1300 feed- 
ing) and pieces of larger pellets (from the treatment feeding at 1400-1500) in the bottom bowl. Significant differences in the frequency of regurgitation between treatment and control fish each day was determined by the Fisher exact test.

Because Lagodon quickly learned to avoid consuming our "larval mimic" (see Results), long-term fitness consequences of consuming didemnins could not be determined for Lagodon. In an attempt to minimize problems associated with learned aversion, we used a neurologically simple consumer, the sea anemone Aiptasia pallida, which we found to be incapable of learned food aversion (see results of both the shortand long-term assays described below). Aiptasia feeds by capturing small organisms such as zooplankton and invertebrate larvae from the water column and, when in photically appropriate environments, via photosynthesis of symbiotic zooxanthellae (Ruppert and Fox 1988). Using Aiptasia was therefore ecologically appropriate because sea anemones, as well as other benthic particle feeders, can be tremendously abundant in benthic habitats (Glynn 1973) and are known to prey heavily on invertebrate larvae (reviewed by Young and Chia 1987). Although Aiptasia and Trididemnum solidum overlap geographically throughout the Caribbean, they do not co-occur in the same habitats; Aiptasia is commonly found in shallow protected bays and in mangrove swamps (Sterrer 1986; N. Lindquist, personal observation) rather than on reefs where Trididemnum solidum occurs. Again, we minimized the chance of prior consumer experience with didemnins by using Aiptasia collected from the temperate waters of North Carolina.

\section{Long-term feeding assay}

For our long-term (32 d) assay, we collected three adjacent anemones from each of 20 locations and used these as 20 blocks for our three separate treatments. Because Aiptasia pallida commonly reproduces vegetatively by breaking off small portions of its base to produce daughter clones (Ruppert and Fox 1988; N. Lindquist, personal observation), this blocking procedure allowed us to minimize variance due to genetic or phenotypic differences among anemones.

Anemones were collected from floating docks in Morehead City, North Carolina in May 1992 by prying off an oyster covered with Aiptasia pallida at each of 20 sites separated by at least $3 \mathrm{~m}$. Three adjacent anemones of similar size were removed from an oyster shell, placed on a smooth, slightly inclined surface for $5 \mathrm{~min}$ to drain off expelled water, and then weighed individually. The consistent dry mass to wet mass ratio measured for 15 anemones $\left(r^{2}=0.88\right)$ indicated that the measured wet mass of anemones provided a good approximation of their size. Anemones were then placed in separate $100-\mathrm{mL}$ plastic bowls arranged in front of three adjacent windows in 20 rows. Each row of three bowls contained the anemones within a block and as- signment of the three treatments within each row was randomized. Because light conditions among bowls varied temporally from minutes, to hours, to days, we made no attempt to quantify light levels for each replicate; however, because we interspersed treatments throughout the entire array (each of the 20 rows contained one replicate of each of the three treatments) variance in rates of growth and asexual reproduction arising from differing light levels for each bowl should be equal for all three treatment groups. Seawater in each bowl was changed daily at 0800 and 1800 . Water temperatures during this assay ranged between $18^{\circ}$ and $21^{\circ} \mathrm{C}$.

In this long-term assay, all anemones were fed a 16.8-mg pellet of commercial fish food (Hikari Cichlid Bio-Gold -"mini"-sized pellets) each day between 0830 and 0900. In addition to this major feeding, accounting for $>98 \%$ of daily food intake, there was an additional feeding between 1500 and 1600 that comprised our various treatments. Treatments consisted of: (1) anemones receiving a single squid pellet (= "larval mimic") as described above (accounting for $1.8 \%$ of total daily food intake by dry mass), (2) anemones receiving a squid pellet that did not contain didemnins (i.e., the control), and (3) anemones receiving no additional feeding. Because the didemnins caused Aiptasia to regurgitate the "larval mimic" (usually within $1 \mathrm{~h}$ ), but the anemones did not learn to avoid ingesting them when offered, we felt this assay could potentially identify longer term consequences of ingesting didemnins, even though regurgitation limited the anemone's exposure to the compounds. Because of this regurgitation by treatment anemones, we added the additional control group, i.e., (3) above, that did not receive a squid pellet to account for the nutritional value lost due to regurgitation of the squid pellet. Squid pellets were offered to anemones daily, except days 17, 24, and 27 when no squid pellets were offered, and day 31 when anemones that had been receiving a treatment squid pellet were given an undefended (i.e., control) squid pellet to determine if they had "learned" to respond (i.e., regurgitate) to some characteristic of the squidbased food other than the presence of the didemnins.

After placing a squid pellet on an anemone's tentacles, we recorded whether or not the anemone ingested the pellet, and if ingested, whether the pellet was regurgitated within $1 \mathrm{~h}$. If regurgitation occurred, the pellet was immediately removed. Using the Fisher exact test, we analyzed for significant differences between the number of treatment and control anemones (1) ingesting the squid pellet and (2) subsequently regurgitating it on each day that squid pellets were offered. Throughout the experiment, we periodically recorded the number of daughter clones that had budded off each adult (squid pellets were offered only to the adult anemone in each bowl). On the final day of the experiment (day 32), we recorded the total number of clones pres- 
ent in each bowl and separately weighed each adult anemone, and its pooled clones.

Our a priori predictions for the results of this assay were (1) that didemnin consumption would reduce anemone growth and vegetative reproduction and (2) that consumption of squid pellets would not contribute significantly to anemone growth and reproduction. Prediction number 1 is based on the significantly lower rate of clone production observed previously among anemones ingesting didemnins in the short-term assay described below and the emetic response of Lagodon ingesting small quantities of didemnins (see Results). Because squid pellets were such a small portion of the control anemone's total ingested diet and photosynthate exuded by zooxanthellae within anemones likely reduced the squid pellet's contribution even more, we predicted that consumption of squid pellets by control anemones would not enhance their growth and reproduction relative to anemones not receiving squid pellets. Using paired-sample $t$ tests, which are an appropriate means of testing planned comparisons between two groups in an experiment with multiple treatments (Sokal and Rohlf 1981: 233), we analyzed for significant differences between treatment anemones ingesting squid pellets with didemnins vs. control anemones ingesting squid pellets without didemnins and between control anemones ingesting normal squid pellets vs. anemones not receiving squid pellets in: (1) the change in wet mass of adult anemones, (2) the total change in wet mass of adults plus clones, (3) the cumulative number of clones produced by each adult anemone, and (4) the average wet mass of daughter clones. To control for the increased probability of Type I error associated with these multiple $t$ tests, the $\alpha$ level was adjusted to $\alpha^{\prime}=0.0253$ using the Dunn-Sidak method $\left[\alpha^{\prime}=1-\right.$ $(1-\alpha)^{1 / k}$ where $k$ is the number of comparisons (Sokal and Rohlf 1981: 242)].

To assess the rates (as opposed to ending totals) at which the different treatments were producing new clones, we regressed the mean number of clones against day of the experiment and compared the slopes of these regression lines using an analysis of covariance (ANCOVA, SYSTAT) followed by a multiple comparison test using A matrices (SYSTAT 1992:238).

\section{Short-term feeding assay}

Before conducting the experiment described above, we ran a preliminary experiment for $8 \mathrm{~d}$ where two groups of 29-30 anemones each were fed a squid pellet ( $3.5 \mu \mathrm{L}=0.3 \mathrm{mg}$ dry mass) with or without didemnins (treatment and control, respectively) each morning on days 1-7 followed 6-8 h later by a piece of fish flesh (as opposed to the commercial fish food provided in the long-term assay) $\approx 8-9 \mathrm{mg}$ dry mass. On day 8 , treatment anemones were offered an undefended control pellet and not a didemnin-treated pellet. This experiment was conducted when water temperatures ranged from $27^{\circ}$ to $29^{\circ} \mathrm{C}$ and anemones were forming daughter clones at a much faster rate than in the longer term assay. This experiment differed from the one described above in that (1) there was no treatment that did not receive a squid pellet, (2) the exact mass of the major food item (the fish flesh) was not rigorously determined for comparison with the mass of the much smaller squid pellet (it did, however, clearly form the majority of daily food intake-we estimate from the dry mass of similar sized pieces of fish flesh that the squid pellet constituted $\approx 3-4 \%$ of food consumed daily), and (3) treatment and control anemones were not paired by microsite from which they were collected. For this experiment, we also regressed the number of clones against the day of the experiment and compared the slopes of the regression lines using an analysis of covariance (ANCOVA, SYSTAT).

\section{RESULTS \\ Feeding assay with Lagodon}

On day 8 when treatment and control fish were first offered a "larval mimic" with and without didemnins, respectively, $100 \%$ of treatment and $100 \%$ of control fish ingested this food (Fig. 1 top). However, within 2 $\mathrm{h}$ of ingesting a "larval mimic," $100 \%$ of the treatment fish regurgitated a mixture of small food pellets eaten by the fish between 1200 and 1300 and pieces of the didemnin-treated squid pellet; no control fish regurgitated (Fig. 1 bottom). Regurgitation frequencies for treatment and control fish on day 8 differed significantly $\left(P=6.5 \times 10^{-9}\right.$, Fisher exact test $)$. On the 9 th d, 13 of 15 treatment and all 15 control fish ingested the "larval mimic" or control pellet, respectively (Fig. 1 top), but again, the number of treatment (13 of 13) vs. control ( 1 of 15$)$ fish regurgitating differed significantly $\left(P=3.7 \times 10^{-7}\right.$, Fisher exact test, Fig. 1 bottom). On day 10 , a significant number of treatment fish (11 of $15, P=2.0 \times 10^{-5}$, Fisher exact test, Fig. 1 top) would not ingest the "larval mimic"; all control fish ingested their food pellet. Of the four treatment fish ingesting the "larval mimic" on day 10, all regurgitated within $2 \mathrm{~h}$. This regurgitation frequency differed significantly $\left(P=1.3 \times 10^{-3}\right.$, Fisher exact test) from that of control fish (Fig. 1 bottom).

\section{Feeding assays with Aiptasia}

On the $27 \mathrm{~d}$ that anemones in the long-term assay were fed squid pellets, $80-100 \%$ of treatment anemones and 95-100\% of control anemones ingested their pellet (Fig. 2 top). The frequency of consuming squid pellets did not differ significantly between treatment and control anemones on any of these days $(P>0.05$, $N=20$, Fisher exact test for each day's data). However, $50-100 \%$ of the anemones receiving didemnin-containing pellets regurgitated the squid pellet within $1 \mathrm{~h}$ of ingestion; no more than $5 \%$ of the control anemones regurgitated the squid pellet (Fig. 2 bottom). The commercial fish food that had been ingested 6-7 h earlier was never regurgitated with the treatment squid pellet. 

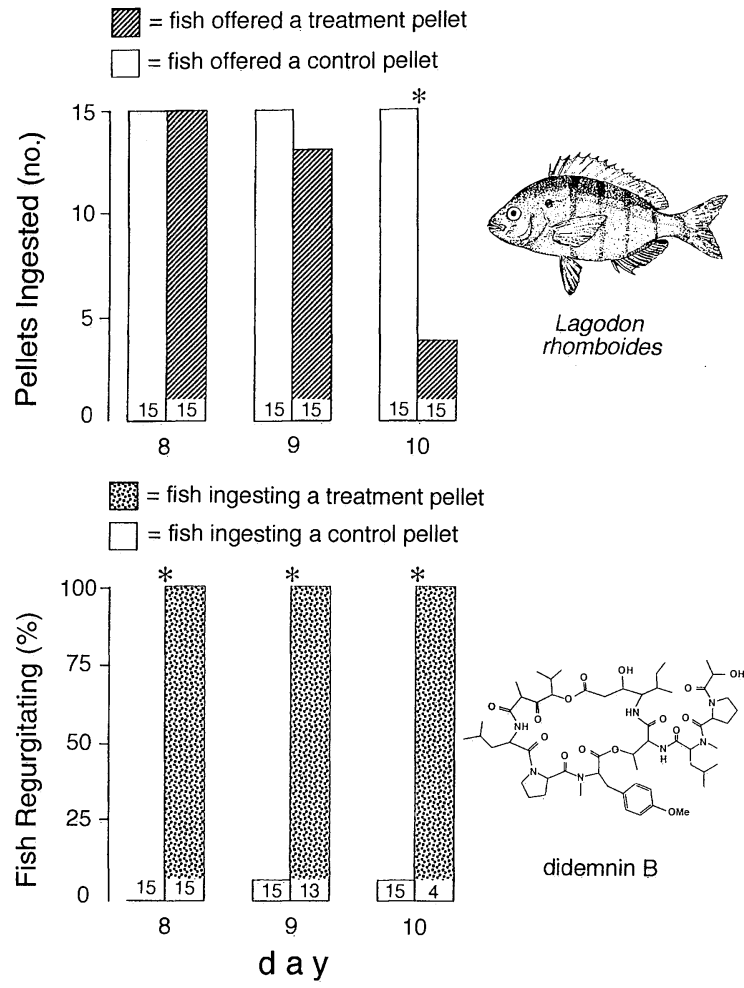

FIG. 1. The number of treatment and control fish (top) ingesting a squid pellet with or without didemnins, respectively, and (bottom) the percent of these fish regurgitating an ingested pellet within $2 \mathrm{~h}$. Treatment and control pellets were offered only on days 8-10. Sample sizes for each assay are shown at the base of the histograms. For each day, significant differences between the number of treatment and control fish ingesting a squid pellet, and regurgitating an ingested squid pellet, are indicated with an asterisk $(P<0.001$, Fisher exact test). The structure of didemnin $\mathrm{B}$, a representative member of the Trididemnum solidum secondary metabolites, is also shown.

Thus, on these $27 \mathrm{~d}$ there was always a significant difference in the frequency of regurgitation between treatment and control anemones $(P=0.0017$ for the day on which the smallest observed difference in regurgitation frequencies occurred, $N=20$ for both treatment and control, Fisher exact test). On day 31, anemones that had been receiving didemnin-containing pellets were offered a control squid pellet; none regurgitated. Thus, didemnins had to be present to elicit an emetic response and there was no learned aversion to the squid-based food.

At the end of the 32-d feeding assay, when the anemones that consumed didemnin-containing squid pellets were compared with those that consumed control squid pellets, it was clear that even this minimal level of didemnin consumption severely affected growth and vegetative reproduction. Didemnin consumption by treatment anemones significantly reduced (1) growth of adults by $82 \%(P=0.013$, paired $t$ test, Fig. $3 \mathrm{~A})$, (2) combined growth of adults and clones by $76 \%(P$
$=0.0022$, paired $t$ test, Fig. 3B), (3) production of clones by $44 \%(P=0.013$, paired $t$ test, Fig. 3C), and (4) the average mass of individual clones by $41 \%$ ( $P$ $=0.0033$, paired $t$ test, Fig. 3D). Anemones fed control squid pellets and those fed no squid pellets did not differ significantly in any of these parameters $(P>$ 0.35 for all comparisons, paired $t$ tests, Fig. 3A-D). Thus, the squid pellet supplement was nutritionally trivial, and treatment anemones were negatively affected by the compounds rather than by regurgitationinduced loss of nutrition contained in the squid pellet. Additionally, regurgitation of the treatment squid pellet never resulted in the loss of the main meal ingested 67 and 14-16 h, respectively, before the squid pellets in the long-term and short-term assays.

In our preliminary 8-d assay, effects of didemnins on feeding and regurgitation were similar to those in our long-term assay. Seventy-three to $100 \%$ of the treatment anemones ingested the treated squid pellet and $60-100 \%$ of these regurgitated within $1 \mathrm{~h}$ (Fig. 4). One hundred percent of control anemones ingested
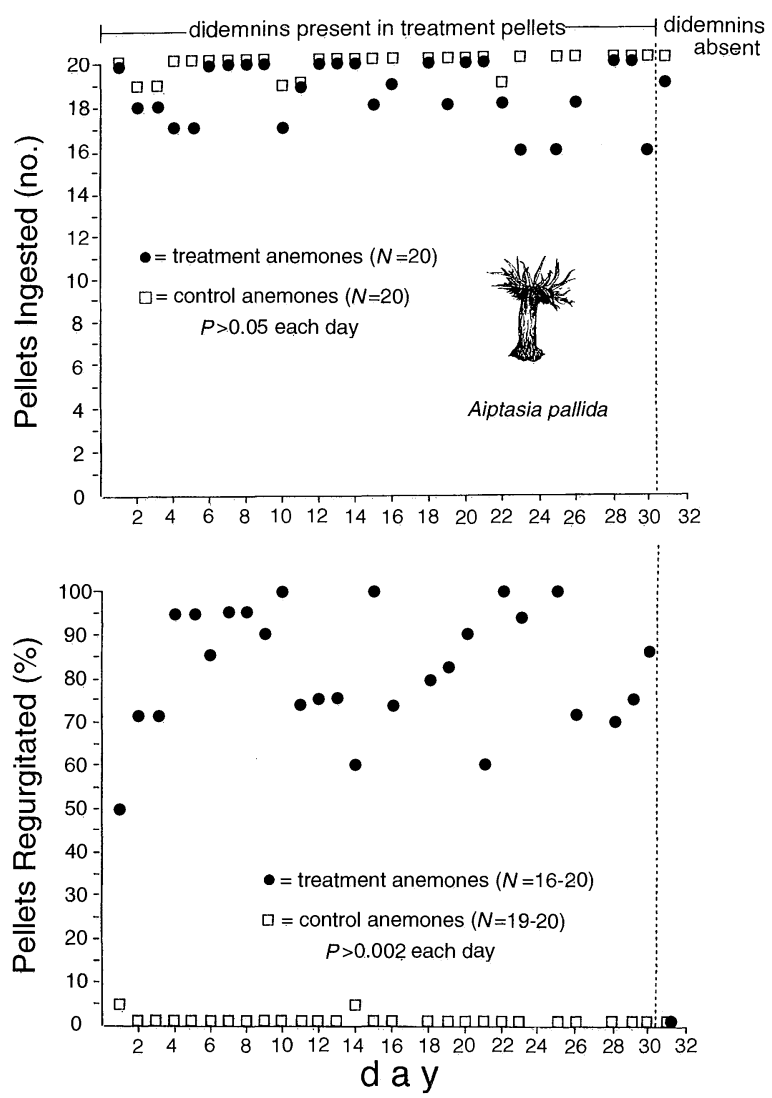

FIG. 2. For each day, the number of treatment and control anemones (top) ingesting a squid pellet with (-) or without ( $\square$ ) didemnins, and (bottom) the percent of these anemones regurgitating the pellet within $1 \mathrm{~h}$. For each day, significant differences between the number of treatment and control anemones ingesting a squid pellet, and regurgitating an ingested squid pellet were determined by the Fisher exact test. 


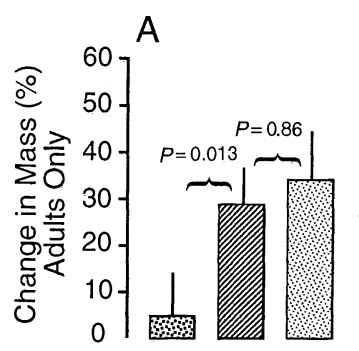

Anemones Receiving:
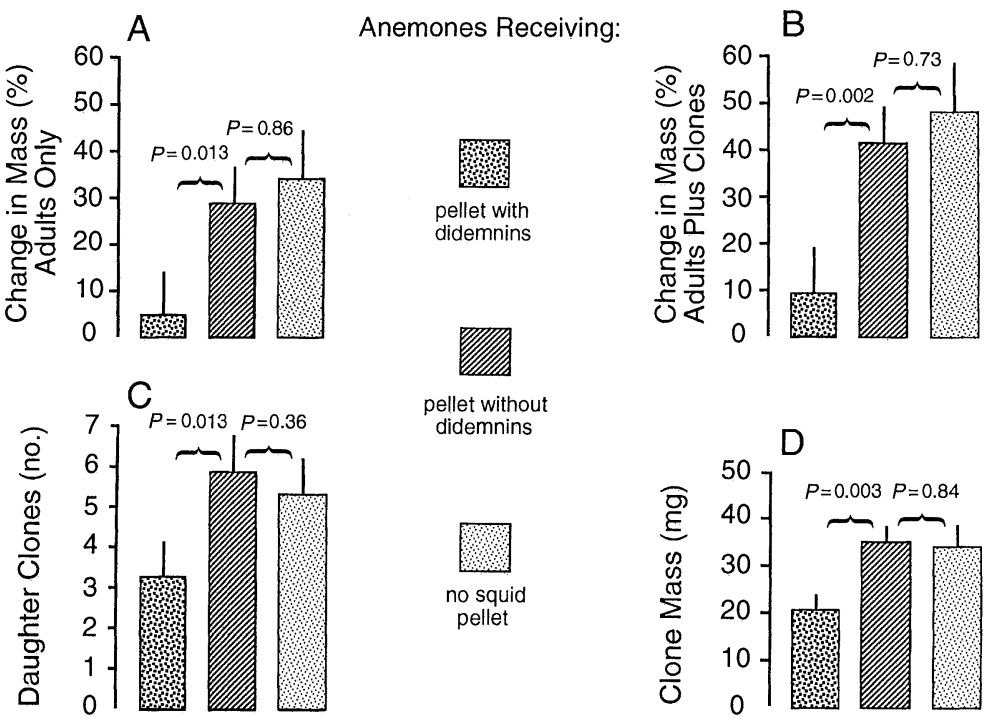

petrins

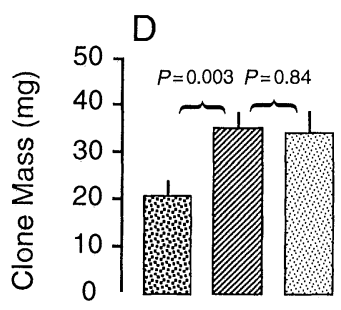

FIG. 3. The increase observed for (A) mass of adult anemones, (B) mass of adults plus daughter clones, (C) the mean number of daughter clones produced by day 32, and (D) the average mass of daughter clones among the three treatments. $N=20$ for each treatment. Error bars show $+1 \mathrm{sE}$. Brackets connect treatments that were compared for significant differences using paired-sample $t$ tests; the $P$ value for each comparison is given above the bracket. The adjusted level of significance for these comparisons is $\alpha^{\prime}=0.0253$.

their squid pellet but $<4 \%$ of these regurgitated. The bolus of digested fish flesh, derived from the meal consumed the previous afternoon, was nearly always egested (as is normal for animals without an anus) by the time a squid pellet was offered the following morning, thus premature egestion of the fish flesh did not occur among treatment anemones. Consumption frequencies between treatment and control anemones did not differ significantly on days $1,2,4,6$, and 8 , but did differ significantly on days 3,5 , and 7 of the short-term assay $(P<0.026$, Fisher exact test, $N=29-30$, Fig. 4$)$. The larger sample size for the short-term assay $(N=29-30$ vs. $N=20$ for the long-term assay) likely increased the power of the statistical test enough so that consumption frequencies between treatment and control anemones occasionally differed significantly in the short-term assay but not the long-term one. In the longterm assay, there was a nonsignificant trend toward decreased consumption of the treatment pellets on days 23,25 , and 30 . On day 8 of the short-term assay, anemones that had been receiving didemnin-containing pellets were offered a control pellet; none regurgitated (Fig. 4).

Fig. 5 shows the rates at which anemones among the various treatments produced new daughter colonies. In the short-term assay run at summer seawater temperatures for North Carolina $\left(27^{\circ}-29^{\circ} \mathrm{C}\right.$, Fig. 5A) treatment anemones produced daughter clones at a significantly slower rate than did control anemones $(P=0.0005$, ANCOVA, Table 1). In the long-term assay run at cooler temperatures $\left(18^{\circ}-21^{\circ} \mathrm{C}\right.$, Fig. 5B) daughter clones also were produced at a significantly slower rate by anemones consuming didemnin-containing squid pel- lets than by anemones receiving a control squid pellet or no squid pellet $(P=0.002$, ANCOVA followed by a multiple comparison test using A matrices, Tables 2 and 3). Rates of daughter clone production did not differ between anemones fed control squid pellets and those not receiving a squid pellet (Fig. 5B).

\section{DISCUSSION}

Demonstrations that ingesting ecologically realistic doses of secondary metabolites can significantly reduce consumer fitness are critical for distinguishing between fitness-based (i.e., compounds being harmful) vs. tastebased (i.e., compounds tasting bad but not being harmful) mechanisms involved in consumer recognition and avoidance of chemically defended prey. Results of our study show that consuming even small amounts of chemically defended prey, similar to, or much less than, levels that some generalist marine planktivores might consume, can produced large negative effects on their fitness. Chemically defended food fed to Aiptasia pallida, as $\approx 1.8 \%$ of the daily diet, reduced growth by $\approx 80 \%$ and asexual reproduction by $\approx 40 \%$ (Figs. 3 and $5)$. In the long-term assay at water temperatures of $18^{\circ}-$ $21^{\circ} \mathrm{C}$, a negative effect of didemnin consumption on budding rate became apparent after 2-3 wk (Fig. 5B). However, in our preliminary 8-d assay (Fig. 5A), didemnin's effect on asexual reproduction became apparent after only 2-4 d of consuming chemically defended "larvae." The higher water temperatures during this assay, $27^{\circ}-29^{\circ} \mathrm{C}$, commonly occur during summer months when many benthic invertebrates in coastal North Carolina release the greatest number of larvae (Sutherland 1981), and these temperatures are the norm 


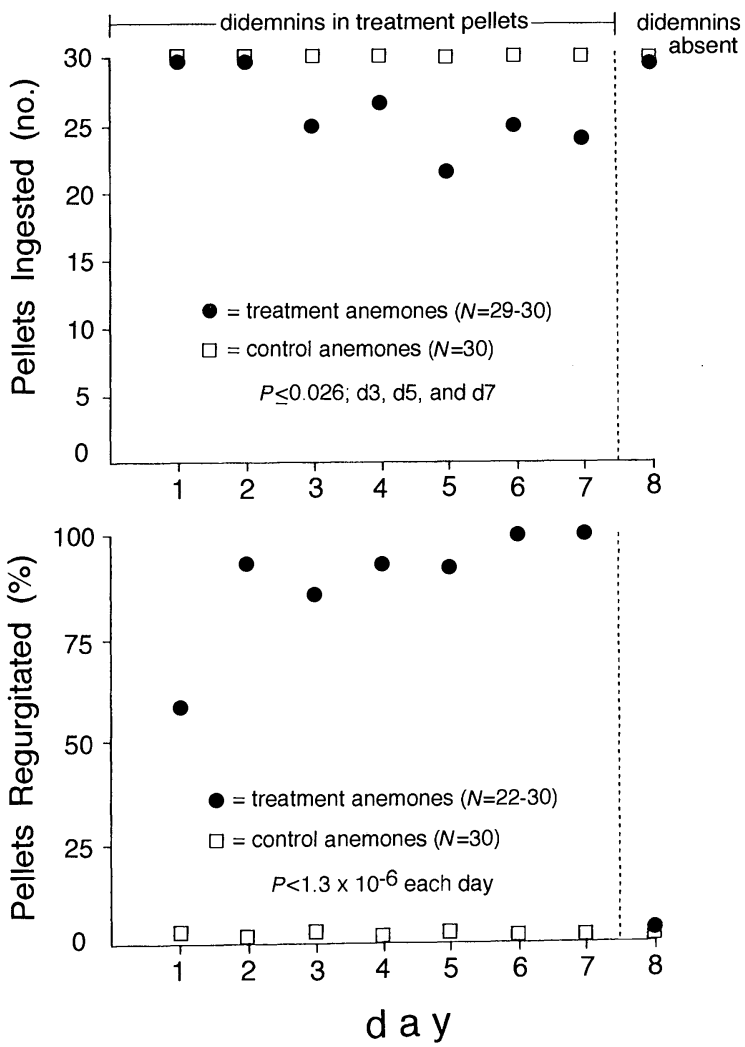

Fig. 4. For each day, the number of treatment and control anemones (top) ingesting a squid pellet with () or without $(\square)$ didemnins, and (bottom) the percent of these anemones regurgitating the pellet within $1 \mathrm{~h}$. For each day, significant differences between the number of treatment and control anemones ingesting a squid pellet, and regurgitating an ingested squid pellet, were determined by the Fisher exact test. for many tropical mangrove and lagoonal areas where Aiptasia pallida are common. The dramatic decreases in fitness that were caused by consuming foods containing didemnins could clearly select for consumers that recognize and reject prey containing these compounds, even when they form a very small portion of the consumer's diet.

Learned food aversion prevented us from identifying longer term physiological consequences for fishes consuming small quantities of didemnin-containing prey; however, results of the feeding assay with Lagodon raise several important points relevant to consumer recognition and avoidance of chemically unsuitable prey. These compounds are apparently not avoided by fishes simply because they "taste" bad, as evidenced by the high rate at which fish consumed didemnin-containing foods on the first $2 \mathrm{~d}$ that they were offered. The didemnins are, however, physiologically disruptive, i.e., they cause Lagodon to regurgitate. This resulted in a significant loss of food, even food ingested 3-4 h prior to regurgitation. Such losses might slow rates of growth, maturation, or reproduction.

Although some studies have focused on the consequences of exposure to, or ingestion of, toxic dinoflagellates on higher trophic levels [e.g., herbivorous copepods (Huntley et al. 1986), fishes (White 1981, Davin et al. 1986, 1988, Magnelia et al. 1992) and sea otters (Kvitek et al. 1991)], assays evaluating the consequences of consuming marine secondary metabolites of benthic macroalgae and invertebrates are rare. At $1 \%$ of the total diet dry mass, a diterpenoid from a brown seaweed reduced growth of an omnivorous fish (Hay et al. 1987), and at $\approx 5.0-5.5 \%$ of total food dry mass, brown algal phlorotannins did not affect Australasian snails or sea urchins (Steinberg and Van Al-
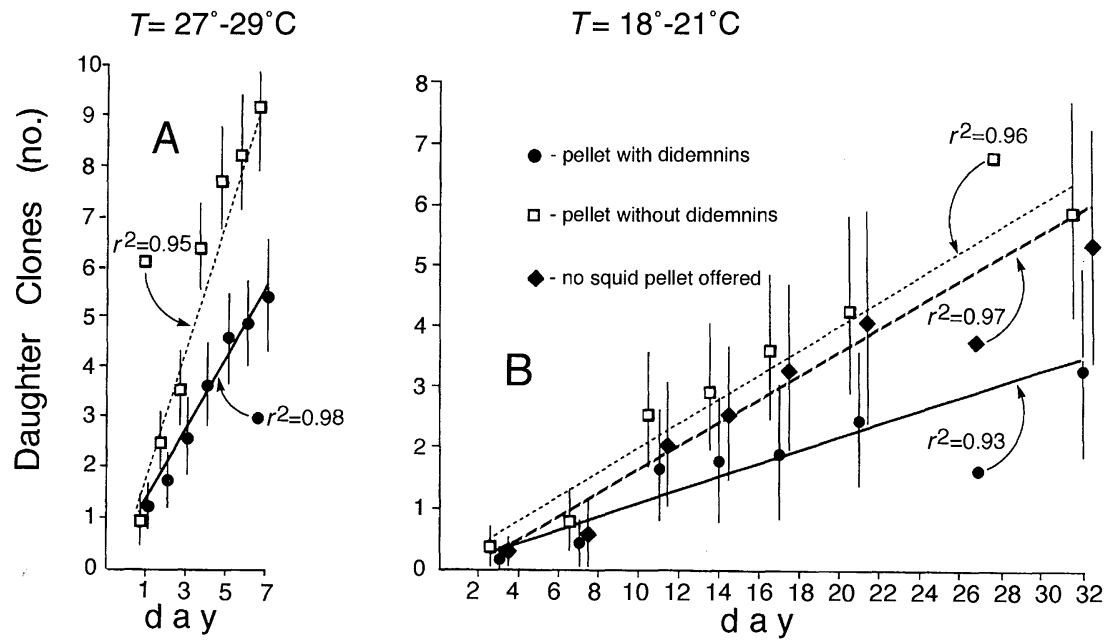

FIG. 5. The production rate of daughter clones among anemones in (A) the short-term (8 d) assay and (B) the long-term (32 d) assay. Error bars show the $95 \%$ confidence interval for each mean. For both assays the mean rate of daughter clone (= buds) production was significantly lower for treatment anemones consuming didemnins vs. controls [ANCOVA, followed by multiple comparison tests using A matrixes for (B) the long-term assay]. 
TABLE 1. Results of the analysis of covariance (ANCOVA) for the regression of the mean number of daughter clones against the day of the experiment between the two treatment groups of anemones in the short-term experiment. The $P$ value for the interaction term, treatment $X$ day (shown in bold type in the table), determines if the rates of daughter clone production ( $=$ slope of the regression lines) differ significantly between treatments.

\begin{tabular}{lccccc}
\hline \hline \multicolumn{1}{c}{$\begin{array}{c}\text { Source of } \\
\text { variation }\end{array}$} & df & ss & MS & $F$ & $P$ \\
\hline Treatment & 1 & 0.260 & 0.260 & 1.057 & 0.328 \\
Day & 1 & 63.3 & 63.3 & 257.1 & $\ll 0.0001$ \\
Treatment $\times$ Day & $\mathbf{1}$ & $\mathbf{6 . 2 9}$ & $\mathbf{6 . 2 9}$ & $\mathbf{2 5 . 5}$ & $\mathbf{0 . 0 0 0 5}$ \\
Residual & 10 & 2.46 & 0.246 & & \\
\hline
\end{tabular}

tena 1992). In a similar experiment, survival of juvenile gastropods (Strombus costatus) was reduced by $45-$ $100 \%$ when fed on algal diets treated with various green algal terpenes at $1 \%$ of diet dry mass (Paul and Fenical 1986). These experiments assessed the effects of prey secondary metabolites on consumers that were limited to eating nothing but compound-containing food. Among these studies, only Hay et al. (1987) limited food consumption by each control to that ingested by its paired treatment. Although concentrations of secondary metabolites used in these assays may approximate those found in the algae from which they were isolated, these assays were almost certainly ecologically unrealistic because generalist herbivores would rarely, if ever, eat a single prey item (and thus its secondary metabolites) for a day, much less for the weeks that these assays were run.

In our long-term assay with the sea anemone Aiptasia pallida, food containing secondary metabolites comprised only $1.8 \%$ (as opposed to $100 \%$ ) of the total ingested diet. Additionally, because treatment pellets were rapidly regurgitated, anemones may have minimized their exposure to didemnins and absorbed less compounds than they consumed. If we assume that all didemnins in the pellets were absorbed by the anemones (probably an unrealistic assumption), and we calculate the didemnin concentration as a proportion of the total dry mass of food ingested each day, then these compounds constituted only $0.04 \%$ of the mass of food eaten by the treatment anemones. Thus, previous tests (Paul and Fenical 1986, Hay et al. 1987, Steinberg and Van Altena 1992) used metabolite concentrations that were at least 23-128 times higher than those used in our long-term assay.

Particulate feeders, such as sea anemones and other benthic cnidarians, consume a wide variety of food types, but tunicate larvae can be common in their guts. As examples, field investigations found that the sea anemone Metridium senile and colonies of the octocoral Alyconium siderium consumed on average 56 and 99 ascidian larvae, respectively, during a $24-\mathrm{h}$ period (Sebens and Koehl 1984). The amount of didemnins anemones ingested in our assays would have been similar to consuming only 15 Trididemnum solidum larvae/ d or only $15-27 \%$ as many larvae as the two particulate feeders studied by Sebens and Koehl (1984) consumed under field conditions.

Because Trididemnum solidum (van Duyl et al. 1981), as well as other colonial ascidians (reviewed by Svane and Young 1989), can release larvae continuously throughout the year and larvae of colonial ascidians often disperse no more than several metres from the adult (Olson 1985, Grosberg and Quinn 1986, Olson and McPherson 1987, Davis and Butler 1989, Stoner 1990, Bingham and Young 1991), predators in the vicinity of these invertebrates may consistently encounter these chemically defended larvae. These encounters may occur frequently enough to enhance selection for rejection of chemically defended larvae. Because chemically unpalatable larvae commonly survive attacks by planktivorous fishes (Young and Bingham 1987, N. Lindquist, personal observation) and benthic cnidarians (N. Lindquist, personal observation), group selection or kin selection need not be invoked to explain the evolution of chemical defenses in larvae, although, for species with short distance larval dispersal, kin selection could facilitate the evolution of chemical defenses in all life stages.

Both fishes and benthic particle-feeders, such as anemones, are reported to consume invertebrate larvae

TABLE 2. Results of the analysis of covariance (ANCOVA) for the regression of the mean number of daughter clones against the day of the experiment among the three treatment groups of anemones in the long-term assay. The $P$ value for the interaction term, treatment $\times$ day (shown bold type in the table), determines if the rate of daughter clone production (= slopes of the regression lines) differed significantly among the three treatments.

\begin{tabular}{lccccr}
\hline \hline \multicolumn{1}{c}{$\begin{array}{c}\text { Source of } \\
\text { variation }\end{array}$} & df & ss & MS & $F$ & $P$ \\
\hline Treatment & 2 & 0.073 & 0.036 & 0.271 & 0.77 \\
Day & 1 & 44.4 & 44.4 & 331.3 & $\mathbf{1 . 2 8}$ \\
Treatment $\times$ Day & $\mathbf{2}$ & $\mathbf{2 . 5 5}$ & 0.001 & $\mathbf{0 . 0 0 2 1}$ \\
Residual & 15 & 2.01 & 0.134 & & \\
\hline
\end{tabular}


TABlE 3. Results of A-matrix multiple comparison tests for the ANCOVA results obtained for the long-term (32 d) experiment. These tests identified treatment groups of anemones that differed significantly in their rates of daughter clone production. The adjusted level of significance for these pairwise comparisons $(\alpha=0.017)$ was determined by dividing 0.05 by the number of comparisons (3).

\begin{tabular}{|c|c|c|c|c|c|}
\hline Pairwise comparison & $\mathrm{df}$ & SS & MS & $F$ & $P$ \\
\hline $\begin{array}{l}\text { Anemones receiving didemnins } \times \text { those re- } \\
\text { ceiving a control pellet }\end{array}$ & 1 & 2.15 & 2.15 & 16.1 & 0.001 \\
\hline $\begin{array}{l}\text { Anemones receiving didemnins } \times \text { those re- } \\
\text { ceiving no squid pellet }\end{array}$ & 1 & 1.64 & 1.64 & 12.3 & 0.003 \\
\hline $\begin{array}{l}\text { Anemones receiving a control pellet } \times \text { those } \\
\text { receiving no squid pellet }\end{array}$ & 1 & 0.035 & 0.035 & 0.259 & 0.62 \\
\hline
\end{tabular}

(reviewed by Young and Chia 1987). Thus, knowledge of how larval secondary metabolites affect the fitness and ultimately prey preferences of these predators is important because predation on larvae can dramatically affect the distribution and structure of invertebrate populations (Gaines and Roughgarden 1987, Olson and McPherson 1987). For fishes, learned food aversion certainly provided an effective means to avoid ingestion of chemically defended prey (Fig. 1). For consumers that are too "stupid" to learn to avoid ingesting a chemically defended prey (e.g., Aiptasia pallida, Fig. 2 ), reduced consumer fitness resulting from consumption of even small numbers of chemically defended "larvae" (Figs. 3 and 5) could select for individuals that innately avoid these prey. This will allow predation to select for physiologically potent secondary metabolites in marine larvae. To our knowledge, this is the first demonstration that marine consumers ingesting very small quantities of chemically defended prey may experience significant depressions in fitness. Thus feeding mistakes resulting in consumption of chemically unsuitable prey may severely reduce a consumer's fitness relative to individuals that sense and avoid prey items containing chemical defenses.

\section{ACKNOWLEDGMENTS}

Support was provided by NSF grants OCE 89-15304 (N Lindquist), OCE 89-11872, and 92-02847 (M.E. Hay), and by a Global Change Distinguished Postdoctoral Fellowship (N. Lindquist) sponsored by the U.S. Department of Energy, Office of Health and Environmental Research, and administered by the Oak Ridge Institute for Science and Education. Comments by Greg Cronin, Jim Estes, Beth Irlandi, David Lodge, Joe Pawlik, Charles Peterson, and two anonymous reviewers improved the manuscript. We thank Phil Levin for assistance with statistical analyses.

\section{Literature Cited}

Ayling, A. L. 1980. Patterns of sexual and asexual reproduction and recruitment in some subtidal marine demospongia. Biological Bulletin 158:271-282.

Bak, R. P. M., J. Sybesma, and F.C. van Duyl. 1981. The ecology of the tropical compound ascidian Trididemnum solidum. II. Abundance, growth and survival. Marine Ecology Progress Series 6:43-52.

Bernays, E. A. 1989. Host range in phytophagous insects: the potential role of generalist predators. Evolutionary Ecology 3:299-311.

- 1991. Relationship between deterrence and toxicity of plant secondary compounds for the grasshopper Schis- tocerca americana. Journal of Chemical Ecology 17:25192526.

Bernays, E. A., and R. Chapman. 1987. The evolution of deterrent responses in plant-feeding insects. Pages 159-173 in R. F. Chapman, E. A. Bernays, and J. G. Stoffolano, Jr., editors. Perspectives in chemoreception and behavior. Springer-Verlag, New York, New York, USA.

Bernays, E. A., and M. Cornelius. 1992. Relationship between deterrence and toxicity of plant secondary compounds for the alfalfa weevil Hypera brunneipennis. Entomologia Experimentalis et Applicata 64:289-292.

Bingham, B. L., and C. M. Young. 1991. Larval behavior of the ascidian Ecteinascidia turbinata Herdman; an in situ experimental study of the effects of swimming on dispersal. Journal of Experimental Marine Biology and Ecology 145: 189-204.

Brattsten, L. B. 1992. Metabolic defenses against plant allelochemicals. Pages 176-242 in G. A. Rosenthal and M. R. Berenbaum, editors. Volume II. Herbivores: their interactions with secondary plant metabolites. Academic Press, San Diego, California, USA.

Darcy, G. H. 1985. Synopsis of biological data on the pinfish Lagodon rhomboides (Pisces: Sparidae). National Oceanic and Atmospheric Administration Technical Report NMFS 23.

Davin, W. T., C. C. Kohler, and D. R. Tindall. 1986. Effects of ciguatera toxins on the bluehead. Transactions of the American Fisheries Society 115:908-912.

Davin, W. T., C. C. Kohler, and D. R. Tindall. 1988. Ciguatera toxins adversely affect piscivorous fishes. Transactions of the American Fisheries Society 117:374-384.

Davis, A. R. 1988. Effects of variation in initial settlement on distribution and abundance of Podoclavella moluccensis Sluiter. Journal of Experimental Marine Biology and Ecology 117:157-167.

Davis, A. R., and A. J. Butler. 1989. Direct observations of larval dispersal in the colonial ascidian Podoclavella moluccensis Sluiter: evidence for closed populations. Journal of Experimental Marine Biology and Ecology 127:189203

Emery, A. R. 1973. Comparative ecology and functional osteology of fourteen species of damselfish (Pisces: Pomacentridae) at Alligator Reef, Florida Keys. Bulletin of Marine Sciences 23:649-700.

Freeland, W. J., and D. H. Janzen. 1974. Strategies in herbivory by mammals: the role of plant secondary compounds. American Naturalist 108:269-289.

Feeny, P. 1992. The evolution of chemical ecology: contributions from the study of herbivorous insects. Pages 1-44 in G. A. Rosenthal and M. R. Berenbaum, editors. Volume II. Herbivores: their interactions with secondary plant metabolites. Academic Press, San Diego, California, USA.

Gaines, S. D., and J. Roughgarden. 1987. Fish in offshore kelp forest affect recruitment to intertidal barnacle populations. Science 235:479-481. 
Gerhart, D. J. 1984. Prostaglandin A2: an agent of chemical defense in the Caribbean gorgonian Plexaura homomalla. Marine Ecology Progress Series 19:181-187.

Glynn, P. W. 1973. Ecology of a Caribbean coral reef. The Porites reef-flat biotope: Part II. Plankton community with evidence for depletion. Marine Biology 22:1-21.

Grosberg, R. K., and J. F. Quinn. 1986. The genetic control and consequences of kin recognition in larvae of a colonial marine invertebrate. Nature 322:456.

Hay, M. E. 1991. Fish-seaweed interactions on coral reefs: effects of herbivorous fishes and adaptations of their prey. Pages 96-119 in P. F. Sale, editor. The ecology of fishes on coral reefs. Academic Press, San Diego, California, USA.

-1992. The role of seaweed chemical defenses in the evolution of feeding specialization and in the mediation of complex interactions. Pages 93-118 in V. J. Paul, editor. Ecological roles for marine natural products. Comstock, Ithaca, New York, USA.

Hay, M. E., J. E. Duffy, C. A. Pfister, and W. Fenical. 1987 Chemical defense against different marine herbivores: are amphipods insect equivalents? Ecology 68:1567-1580.

Hay, M. E., and W. Fenical. 1988. Marine plant-herbivore interactions: the ecology of chemical defense. Annual Review of Ecology and Systematics 19:111-145.

Hay, M. E., and P. D. Steinberg. 1992. The chemical ecology of plant-herbivore interactions in marine versus terrestrial communities. Pages 371-413 in J. Rosenthal and M. Berenbaum, editors. Herbivores: their interaction with secondary metabolites, evolutionary and ecological processes. Academic Press, San Diego, California, USA.

Hobson, E. S., and J. R. Chess. 1976. Trophic interactions among fishes and zooplankters near shore at Santa Catalina Island, California. United States National Marine Fisheries Service Fishery Bulletin 74:567-598.

Huntley, M., P. Sykes, S. Rohan, and V. Marin. 1986. Chemically-mediated rejection of dinoflagellate prey by the copepods Calanus pacificus and Paracalanus parvus: mechanism, occurrence and significance. Marine Ecology Progress Series 28:105-120.

Ilan, M., and Y. Loya. 1990. Sexual reproduction and settlement of the coral reef sponge Chalinula sp. from the Red Sea. Marine Biology 105:25-31.

Kerfoot, W. C. 1982. A question of taste: crypsis and warning coloration in freshwater zooplankton communities. Ecology 63:538-554.

Kvitek, R. G., A. R. DeGange, and M.K. Beitler. 1991. Paralytic shellfish poisoning toxins mediate feeding behavior of sea otters. Limnology and Oceanography 36:393-404.

Lindquist, N., M. E. Hay, and W. Fenical. 1992. Defenses of ascidians and their conspicuous larvae: adult versus larval chemical defenses. Ecological Monographs 62:547568.

Livingston, R. J. 1984. Trophic response of fishes to habitat variability in coastal seagrass systems. Ecology 65:12581275

Lucas, J. S., R. J. Hart, M. E. Howden, and R. Salathe. 1979. Saponins in eggs and larvae of Acanthaster planci (L.) (Asteroidea) as chemical defenses against planktivorous fish. Journal of Experimental Marine Biology and Ecology 40: $155-165$.

Magnelia, S. J., C. C. Kohler, and D. R. Tindall. 1992. Acanthurids do not avoid consuming cultured toxic dinoflagellates yet do not become ciguatoxic. Transactions of the American Fisheries Society 121:737-745.

Olson, R. R. 1983. Ascidian-prochloron symbosis: the role of larval photoadaptation in midday larval release and settlement. Biological Bulletin 165:221-240.

- 1985. The consequences of short-distance dispersal in a sessile marine invertebrate. Ecology 66:30-39.

Olson, R. R., and R. McPherson. 1987. Potential vs. realized larval dispersal: fish predation on larvae of the ascidian Lissoclinum patella (Gottschaldt). Journal of Experimental Marine Biology and Ecology 110:245-256.

Paul, V. J., editor. 1992. Ecological roles of marine natural products. Comstock, Ithaca, New York, USA

Paul, V. J., and W. Fenical. 1986. Chemical defense in tropical green algae, order Caulerpales. Marine Ecology Progress Series 34:157-169.

Pawlik, J. R. 1993. Marine invertebrate chemical defense. Chemical Reviews 93:1911-1922.

Purcell, J. E. 1981. Selective predation and caloric consumption by the siphonophore Rosacea cymbiformis in nature. Marine Biology 63:283-294.

Reiswig, H. M. 1973. Population dynamics of three Jamaican demospongiae. Bulletin of Marine Science 23:191-226.

Rosenthal, G. A., and M. E. Berenbaum, editors. 1992. Herbivores: their interactions with secondary plant metabolites. Volume II. Academic Press, San Diego, California, USA.

Ruppert, E., and R. Fox. 1988. Seashore animals of the southeast. University of South Carolina Press, Columbia, South Carolina, USA.

Sebens, K. P., and M. A. R. Koehl. 1984. Predation on zooplankton by the benthic anthozoans Alcyonium siderium (Alcyonacea) and Metridium senile (Actiniaria) in the New England subtidal. Marine Biology 81:255-276.

Slansky, F., Jr. 1992. Allelochemical-nutrient interactions in herbivore nutritional ecology. Pages $135-174$ in G. A. Rosenthal and M. R. Berenbaum, editors. Herbivores: their interactions with secondary plant metabolites, Volume II. Academic Press, San Diego, California, USA.

Sokal, R. R., and F. J. Rohlf. 1981. Biometry. W. H. Freeman, San Francisco, California, USA.

Steinberg, P. D., and I. Van Altena. 1992. Tolerance of marine invertebrates herbivores to brown algal phlorotannins in temperate Australasia. Ecological Monographs 62:189222.

Sterrer, W. 1986. Marine fauna and flora of Bermuda. John Wiley \& Sons, New York, New York, USA.

Stoner, D. S. 1990. Recruitment of a tropical colonial ascidian: relative importance of pre-settlement vs. post-settlement processes. Ecology 71:1682-1690.

Sutherland, J. P. 1981. The fouling community at Beaufort, North Carolina: a study in stability. American Naturalist 118:499-519.

Svane, I., and C. M. Young. 1989. The ecology and behavior of ascidian larvae. Pages 45-90 in M. Barnes, editor. Volume 27. Oceanography and marine biology annual review. Aberdeen University Press, Aberdeen, Scotland.

SYSTAT. 1992. SYSTAT for Windows: statistics. Version 5 edition. SYSTAT, Evanston, Illinois, USA.

Thorson, G. L. 1950. Reproductive and larval ecology of marine bottom invertebrates. Biological Reviews 25:1-45.

van Duyl, F. C., R. P. M. Bak, and J. Sybesma. 1981. The ecology of the tropical compound ascidian Trididemnum solidum. I. Reproductive strategy and larval behaviour. Marine Ecology Progress Series 6:35-42.

White, A. W. 1981. Sensitivity of marine fishes to toxins from the red-tide dinoflagellate Gonyaulax excavata and implications for fish kills. Marine Biology 65:255-260.

Young, C. M., and B. L. Bingham. 1987. Chemical defense and aposematic coloration in larvae of the ascidian Ecteinascidia turbinata. Marine Biology 96:539-544.

Young, C. M., and F.-S. Chia. 1987. Abundance and distribution of pelagic larvae as influenced by predation, behavior, and hydrographic factors. Pages $385-463$ in A. C. 
Giese, J. S. Pearse, and V. B. Pearse, editors. Volume 9. Zelickman, E. A., V. I. Gelfand, and M. A. Shifrin. 1969. Reproduction of marine invertebrates. Blackwell Scientific,

Palo Alto, California and Boxwood Press, Pacific Grove, California, USA.

Growth, reproduction and nutrition of some Barents Sea hydromedusae in natural aggregations. Marine Biology 4: 167-173. 